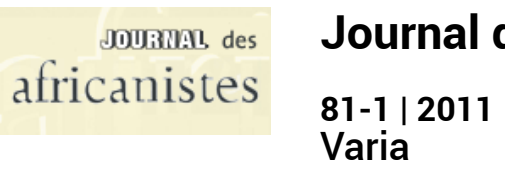

\title{
Comment dansent les institutions : Classes d'âge et rapports de sexe chez les Bassari de Guinée
}

How institutions dance. age and gender among the Bassari of Guinea

\section{Laurent Gabail}

\section{(2) OpenEdition}

\section{Journals}

Édition électronique

URL : http://journals.openedition.org/africanistes/3685

ISSN : 1957-7850

Éditeur

Société des africanistes

Édition imprimée

Date de publication : 1 octobre 2011

Pagination : 9-33

ISBN : 978-2-908948-36-3

ISSN : 0399-0346

Ce document vous est offert par Université Toulouse 2 - Jean Jaurès

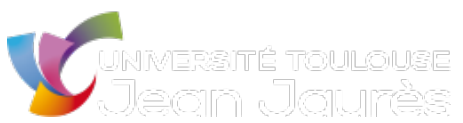

Référence électronique

Laurent Gabail, «Comment dansent les institutions : Classes d'âge et rapports de sexe chez les Bassari de Guinée », Journal des africanistes [En ligne], 81-1 | 2011, mis en ligne le 05 janvier 2016, consulté le 18 février 2019. URL : http://journals.openedition.org/africanistes/3685 
Laurent GABAIL ${ }^{*}$

\title{
Comment dansent les institutions : Classes d'âge et rapports de sexe chez les Bassari de Guinée ${ }^{1}$
}

\begin{abstract}
Résumé
Les Bassari de Guinée organisent périodiquement des danses collectives à l'occasion desquelles les classes d'âge masculines et féminines se rassemblent pour exhiber leurs ornements. L'article cherche à rendre compte des raisons pour lesquelles l'esthétique de la danse semble davantage appréciée en fonction du mouvement des ornements qu'en fonction de celui des corps des danseurs. En sous-évaluant certaines distinctions conventionnelles telles que celles entre ornements et instruments ou entre corps et artefacts, la danse offre aux participants l'expérience simultanée de deux modes d'identification radicalement différents : le premier relève de l'indifférenciation collective des groupes d'âge initiatiques; le second de la place stratégiquement négociée au sein de réseaux relationnels égocentrés.
\end{abstract}

Mots-clés : Danse, rituel, système d'âge, rapports de sexe, ornements, Afrique, Guinée, Bassari.

\section{How Institutions dance. Age and gender among the Bassari of Guinea}

\section{Abstract}

The Bassari of Guinea organize periodically collective dances during which male and female age sets gather to exhibit their ornaments. This article aims at understanding why people seem more incline to define their dancing practice according to the way ornaments move rather than how their body do. By undermining certain conventional distinctions, such as those between ornaments and instruments or between bodies and artefacts, dance provides participants with the simultaneous experience of two radically different modes of identification: one relates to the collective undifferentiation of initiatory age-sets, the other to the ego-centric and strategically negotiated relational networks developed by each individual dancer.

Keywords : Dance, Ritual, Age, Gender, Ornaments, Bassari, Guinea.

\footnotetext{
* CEMAf, musée du quai Branly.

${ }^{1}$ Je tiens à remercier chaleureusement les participant(e)s de l'atelier 'La danse comme objet anthropologique' (CEMAf/Laboratoire d'Anthropologie des Pratiques Corporelles) et du séminaire 'Art et performance' (musée du quai Branly) pour leurs suggestions et remarques, ainsi que Michael Houseman, Magali De Ruyter et François Berthomé pour leur lecture d'une version antérieure de ce texte.
} 
Avril, saison sèche. Récemment rentrés des migrations saisonnières où ils sont partis gagner un peu d'argent dans les villes du Sénégal et de la Guinée, vendant leurs services de récolteurs de vin de palme, d'artisans vanniers, de chasseurs ou encore de pileuses de grain dans des familles peules, les jeunes hommes et femmes bassari ${ }^{2}{ }^{\prime}$ 'affairent. C'est le temps du premier grand rassemblement de la saison sèche, celui de la danse owi. Les danseurs, des trentenaires de la classe d'âge odyar, demandent aux femmes de leur tresser les cheveux afin qu'on puisse y accrocher de grands cimiers en plumes de vautours. Mandatés par les hommes, les jeunes initiés sillonnent le village afin de collecter les ornements de chaque danseur. De leur côté, les jeunes filles d'une vingtaine d'années qui participeront à la danse réparent leurs ceintures de perles, ajustent les décorations de leur porte-bébé et, surtout, surveillent attentivement la préparation de la bière de mil qui devra arriver en quantité au village pour que la fête soit réussie. La raison de cette mobilisation tient à ce que pendant trois semaines chacun des six villages d'un même groupement accueillera tour à tour tous les autres, créant ainsi grâce à la danse un contexte où peuvent se retrouver des gens qui, vivant dispersés, ne se sont pour la plupart d'entre eux pas vus depuis plusieurs mois.

Cet article se propose de décrire et d'analyser ce qui n'apparaît qu'en creux dans cette vignette : la danse. Il trouve son origine dans les commentaires qu'ont pu me faire les Bassari lorsque je tentais de comprendre sur quoi repose leur jugement esthétique. Déjà habitué aux discours obliques ou laconiques qu'engendrent immanquablement des questions frontales, je tâtonnais en essayant de repérer les bons et les mauvais danseurs. Autant mon oreille avait acquis la compétence d'identifier sans trop d'erreurs les bons chanteurs - ce qui me valait, sur ce point, un certain prestige car les Bassari valorisent le chant au plus haut point -, autant mon œil tombait rarement juste. Ou plutôt, je n'étais pas sûr que nous parlions de la même chose lorsque je ne me trompais pas. En effet,

\footnotetext{
2 Les Bassari, qui se nomment eux-mêmes be-liyan (litt. "Ceux de la latérite »), appartiennent au groupe linguistique tenda qui comprend également les Bedik, les Coniagui, les Badyaranké et les Boïn. Les Bassari sont traditionnellement des chasseurs collecteurs qui pratiquaient une agriculture de complément aujourd'hui devenue largement majoritaire. Ils sont environ 15000 et partagent avec des pasteurs semi-nomades peuls un territoire au relief accidenté, de part et d'autre de la frontière entre le Sénégal et la Guinée. Je travaille depuis 2006 en Guinée, dans le groupe de villages dit 6ə-xoré (litt. «ceux du koré», du nom de l'initiation masculine) qui constitue l'un des cinq groupements majoritairement endogames au sein desquels on peut observer une plus grande proximité linguistique et rituelle. Entamée en Guinée par Delacour (1910), l'ethnographie des Bassari s'est poursuivie, à partir du régime politique de Sékou Touré, au Sénégal (groupement 6ane) et a donné lieu depuis le début des années 1960 à de nombreuses publications, principalement de Monique Gessain (1971, 2006) et de Marie-Paule Ferry (1991).
} 
à maintes reprises, mon attention fut recentrée non pas sur les corps des danseurs, mais sur leurs ornements, et cela de manière parfois assez énigmatique : «tu as vu la ceinture de perles? » (avec un geste de la main pour en imiter le mouvement) ; « regarde, je suis sûr qu'il s'agit de la cloche que j'avais prêtée à T. et qu'il m'a dit qu'il avait perdue » (pour souligner le caractère stratégique des échanges d'ornements) ; «Quand nous on dansait owi, fallait voir !» (venant d'un membre d'une classe d'âge ancienne pour souligner les changements ornementaux des modes successives), etc. Ce qui m'avait intrigué sur le terrain est que les Bassari résistent à qualifier directement la qualité du geste dansé pour lui-même. Au contraire, ils le mesurent beaucoup plus volontiers en fonction du mouvement ornemental qui en résulte. On ne parlera ainsi pas du mouvement du bassin provoqué par l'accentuation de la marche alternée de telle danseuse, mais du balancement cadencé des perles qui pendent à sa ceinture. L'essentiel de mon propos consistera à suggérer un cadre qui contribue à rendre intelligibles des énoncés qui m'ont longtemps profondément déconcerté. Pour anticiper le cours de la discussion et synthétiser les commentaires de Bassari, disons que lorsque je formulais des interrogations concernant la danse, mes interlocuteurs me parlaient des ornements, et que lorsque j'ai fini par m'intéresser aux ornements, c'est de relations dont il était question.

Pour saisir toutes les implications de la focalisation qu'opèrent les Bassari sur l'ornementation des corps, il importe tout autant d'identifier certains des aspects formels de la danse que d'envisager le contexte sociologique plus général dans laquelle elle s'inscrit. Etablir un lien entre ces deux échelles d'analyse (l'une interne à la danse, l'autre externe) est l'objet de cet article. Nous verrons qu'au travers de quelques procédés chorégraphiques relativement simples, il est en effet possible de rendre saillants les ornements des danseurs et danseuses et de distinguer hiérarchiquement les groupes en présence. La danse qui fait l'objet de cet article rassemble selon un modèle concentrique trois chaînes de danseurs : un groupe d'hommes à la périphérie et deux groupes de femmes à l'intérieur. Ces trois groupes représentent chacun une classe d'âge, arborent une série d'ornements distinctifs, et exécutent une chorégraphie circulaire sans improvisation possible. La fonction que remplissent les ornements au sein de ce dispositif chorégraphique est non seulement d'indiquer l'appartenance d'un individu à un groupe d'âge masculin ou féminin, mais également de donner à voir les liens que ces différentes classes d'hommes et de femmes entretiennent mutuellement. Loin d'être réductibles à de simples accessoires esthétiques, les ornements doivent donc être appréhendés comme des «indices de relations» (Gell, 1998). L'identification des relations qu'indexent les ornements permet alors d'analyser la danse comme 
un mode ritualisation sous-tendu par des configurations relationnelles qui témoignent d'une forme spécifique (Houseman \& Severi 1994 ; Houseman 2006).

En replaçant la danse dans son contexte sociologique plus général, je souhaite également souligner que si la danse objective les puissantes contraintes classificatoires qui s'exercent selon l'âge et le sexe, elle ne se contente pas de refléter une organisation sociale qui lui préexiste : elle y participe pleinement. Le problème majeur qui se pose est d'apprécier l'efficacité de la danse dans l'émergence des identités collectives que supposent le système de classes d'âge. Prétendre que la danse génère des distinctions d'âge et de sexe est à l'évidence inexact (bon nombre d'autres activités y concourent), admettre qu'elle les exprime est presque trivial. Dans un cas on se situe bien au-delà du degré auquel la danse est opératoire, dans l'autre on reste largement en deçà de ce qui en fait la spécificité. Mon hypothèse est que la danse est un mode de participation particulièrement efficace pour donner à voir la position qu'on occupe dans un système de relations. Dans le cas ici analysé, j'essaierai de montrer que la danse impose un cadre formel puissant à de séquences d'actions collectives et que cela permet de rendre publiquement visible, au travers des ornements qui en constituent l'ancrage matériel, l'institution du système de classes d'âge en tant que telle.

\section{LES AMIES FORMELLES ET LES EPOUSES POTENTIELLES}

Les contextes d'exécution des activités musicales et dansées des Bassari obéissent à des schémas rigoureux (Dehoux \& Gessain 1992). A l'instar des autres danses, la danse $o \tilde{w i}$ intervient à un moment précis de la vie des hommes et des femmes qui l'exécutent, dans une période particulière de l'année, et dans un lieu bien déterminé. Les places de danse sont en effet toujours situées dans le «village de fêtes ». Seuls résident de manière permanente dans ce village abandonné le reste de l'année le chef et sa famille, ainsi que les jeunes garçons et jeunes filles célibataires qui occupent, selon leur appartenance d'âge, deux «maisons communes » (ambofor). Outre la précision du lieu où elles se déroulent, les danses bassari sont invariablement la propriété exclusive de groupes d'âge masculin et/ou féminin, ce qui signifie que chaque individu exécutera, dans un ordre déterminé par son avancée dans le système d'âge, toutes les danses. Appartenir à tel groupe d'âge donne le droit de danser telle danse et, réciproquement, quitter une classe d'âge implique de céder sa danse au groupe suivant.

Hommes et femmes s'ordonnent selon des échelons d'âge dès quinze ans pour les garçons (après l'initiation), et aux environs de douze ans pour 
les filles ${ }^{3}$. Le vocabulaire pour désigner les rapports entre les classes d'âge est emprunté à la nomenclature de parenté. Les membres des classes d'âge consécutives s'appellent réciproquement «pères »/«fils» et «mères »/« filles » et leurs relations sont teintées de distance et de respect. A l'inverse, les membres des classes alternes ont recours au terme d'adresse, neutre et autoréciproque, qui désigne les grands-parents et les petits-enfants et son usage renvoie aux relations à plaisanterie que ces derniers entretiennent. Au terme d'un cycle de six ans, le calendrier rituel connaît une année particulière où tout le système se décale d'un échelon : les classes $\mathrm{C}$ deviennent $\mathrm{D}$, les $\mathrm{D}$ deviennent $\mathrm{E}$, etc. Les femmes suivent un chemin parallèle en changeant synchroniquement de classe avec leurs homologues masculins. Les premiers échelons d'âge féminins sont calqués sur ceux des hommes au point que leurs noms dérivent directement de ceux des échelons masculins: la classe féminine des od-odyar, par exemple, signifie littéralement «celles des » odyar. Le passage d'une classe à une autre implique de s'être acquitté de lourds travaux collectifs imposés à chacun des deux sexes ainsi que, pour les hommes, de subir de leurs «pères » un certain nombre de brimades initiatiques. La logique étant qu'on fait subir à la classe inférieure exactement ce qu'on a subi de la classe supérieure et, réciproquement, qu'on bénéficie de la part de ses «fils » des mêmes prestations (souvent quantifiées en jarres de bière de mil) que celles dont ont bénéficiés en leur temps les «pères ».

Les nouvelles promotions d'initiés s'installent alternativement, par cycle de six ans, dans l'une des deux maisons communes de telle sorte que les nouveaux arrivants investissent un lieu qu'occupent déjà des hommes plus vieux qu'eux de deux cycles. Les fillettes intègrent la maison de leurs camarades masculins un échelon plus tard que les nouveaux initiés, puis suivent un parcours identique qui les distingue de leurs « mères » et de leurs « filles » tout en les assimilant à leurs « grand-mères ». En vertu de cette affiliation résidentielle, la société toute entière se trouve organisée selon un principe dualiste. Les classes alternes sont en effet pensées comme corésidentes et la bipartition des maisons d'âge sépare deux groupes fondés chacun sur des relations transitives : un grand-père de grand-père est un corésident de corésident, donc de manière classificatoire aussi bien un corésident qu'un grand-père, l'ensemble de ces classes alternes formant des parents à plaisanterie. Les relations entre chacune des moitiés résidentielles sont, à l'inverse, fondamentalement asymétriques et l'opposition se formule

\footnotetext{
${ }^{3}$ Je simplifie ici volontairement le système de classes d'âges. Il y a en fait davantage de classes masculines et féminines, à la fois plus jeunes et plus âgées. Pour le système d'âge des Bassari d'Etyolo (Sénégal), plus complexe qu'en Guinée dans les derniers échelons féminins, voir notamment Gessain (1971).
} 
en terme de "pères » et de «fils », d'initiateurs et d'initiés. Idéalement, les hommes épousent les femmes de la classe d'âge immédiatement supérieure à la leur et qui ne résident donc pas dans la même maison commune qu'eux. Il est important de noter que cette relation d'affinité potentielle est désignée par un terme précis qui n'appartient pas à la nomenclature de parenté. Ces individus sont mbanyira les uns pour les autres, ce qui évoque aussi bien cette virtualité de mariage qu'une relation de danse privilégiée. On dit des mbanyira que ce sont ceux qui «dansent ensemble». Cette relation classificatoire entre mbanyira s'oppose à une autre modalité de relation, nommée nyapra - elle aussi spécifique au vocabulaire des catégories d'âge - qui est une relation élective instituée, au moment de l'entrée dans la maison commune, entre une jeune fille de la classe odopalug et l'un de ses camarades d'âge opalug (en moyenne dix ans plus vieux). Si les relations entre nyapra sont des relations de corésidence, de commensalité, d'échanges et éventuellement de partenariat sexuel, celles entre mbanyira sont supposées être l'inverse : maisons distinctes, rapport de honte et d'évitement, etc. Ces deux modalités de relations débordent le cadre villageois et s'appliquent à l'ensemble d'un groupe de villages : un homme (ou une femme) est lié(e) à autant de nyapra qu'il y a de villages, et à toutes les femmes (hommes) des villages voisins qui sont sous ce rapport de classe mbanyira.

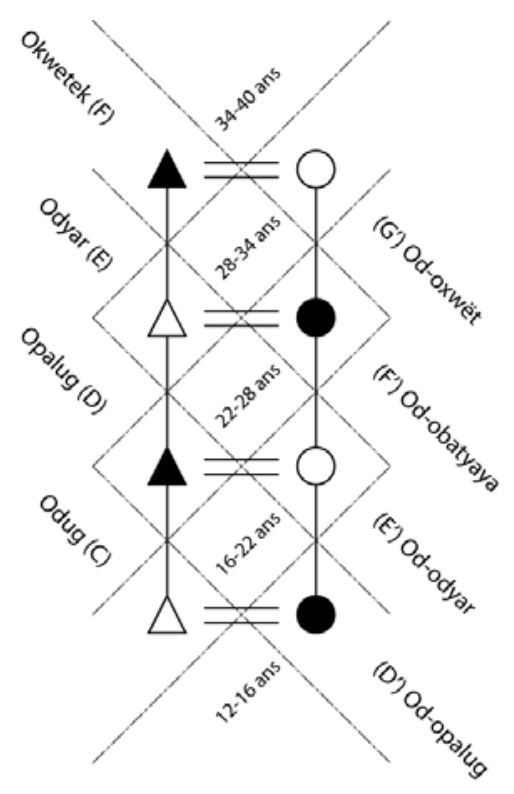

Fig. 1. Système d'âge, affiliation résidentielle et affinité potentielle 
La figure 1 présente sur un même schéma la plupart des classes masculines et féminines (gauche et droite dans la diagonale NO/SE), l'approximation des âges biologiques correspondants (diagonale SO/NE), les appartenances résidentielles (noir et blanc), ainsi que le mariage potentiel (=).

\section{«REGARDE BOUGER L'ORNEMENT! »}

Danse la plus valorisée par les hommes, la danse $o \tilde{w} i$ est aussi celle qui possède l'extension territoriale la plus grande en couvrant l'ensemble des six villages du groupement 6ə-xoré. Elle se déroule chaque année pendant trois semaines en associant les villages par paire : trois jours dans les deux premiers villages la première semaine, trois jours la semaine suivante dans deux autres villages du même groupement, puis trois jours encore dans les deux derniers la troisième semaine. A chacune de ces trois occasions cérémonielles, la danse doit idéalement présenter le moins d'interruptions possibles, ne laissant aux participants que le temps de dormir quelques heures. Son dispositif chorégraphique central rassemble en trois chaînes concentriques de danseurs et danseuses, une classe d'hommes, les odyar (E), et deux classes de femmes, les od-obatyaya (F') et les od-odyar (E'). À ces trois groupes de danseurs et danseuses s'ajoutent une classe d'âge masculine de musiciens tambourinaires, les okwetzk (F), ainsi qu'une chaîne périphérique de danseuses constituée de vieilles femmes (cf.. figure 2).

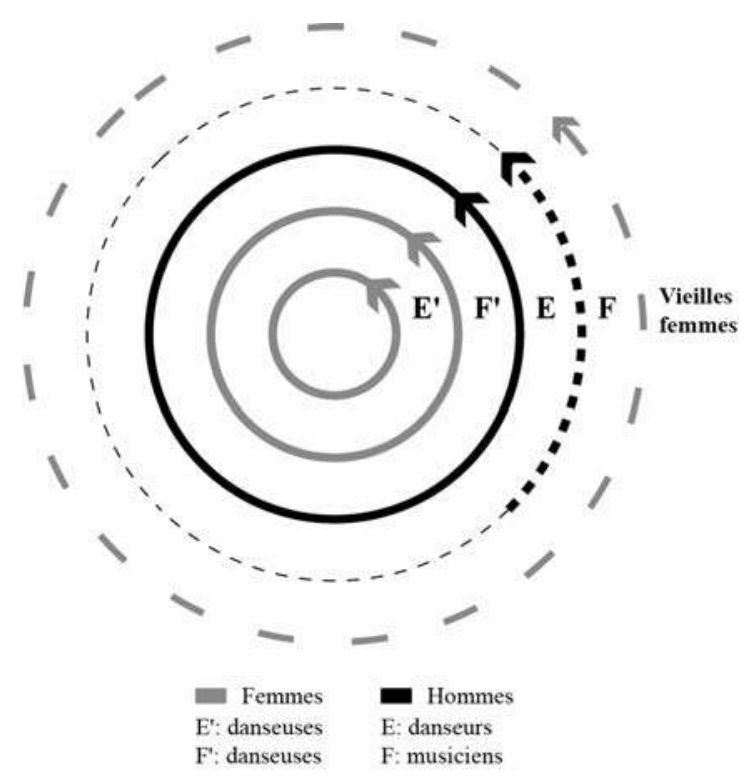

Fig. 2. Organisation spatiale des chaînes de danseurs 
En tant que classes successives (E' et F'), les deux groupes de femmes sont dans un rapport terminologique de «mères » à «filles ». Les hommes (E) dansent donc aussi bien en même temps que leurs mbanyira, les femmes odobatyaya d'une classe immédiatement supérieure à la leur, qu'avec les jeunes femmes de leur classe d'âge, les od-odyar, parmi lesquelles se trouvent les nyapra de chacun d'entre eux. Les chaînes de danseurs sont organisées de telle sorte que les hommes forment un cercle périphérique qui englobe celui des od-obatyaya, ce dernier englobant à son tour celui des ododyar (cf. photogramme 1 page suivante).

Hommes comme femmes affichent une attitude opaque où l'impératif de contrôler l'expression des émotions ne permet pas plus de montrer l'ivresse que la fatigue. Seules quelques vieilles femmes, qui forment une chaîne informelle et bruyante de danseuses peuvent adopter une attitude débridée. Elles portent au dos dans un vieux tissu un bidon rempli de bière de mil parodiant le porte-bébé subtilement décoré qu'affichent les danseuses. Mais la cible privilégiée des vieilles femmes reste les hommes dont elles menacent de couvrir le corps de cendre de bois lorsqu'ils interrompent la danse pour se reposer. Libres également de participer à la danse sans y appartenir, on peut compter de nombreux enfants qui imitent leurs aînés, traversant les chaînes de danseurs pour parfois même se placer en leur centre. Mise en relief par la parodie provocatrice des vieilles femmes et l'imitation libre des enfants, l'idée d'unité de la classe d'âge se laisse facilement saisir par l'uniformité ornementale et chorégraphique que doit afficher chaque classe, chaque ligne de danseurs et de danseuses exécutant exactement les mêmes pas, sans improvisation possible. Le terme qu'on emploie d'ailleurs pour «quitter la danse », «se retirer» (a-syว́fàtá), qui signifie littéralement «faire un trou », et que l'on utilise de manière dérivée dans le travail agricole au sens «d'éclaircir» (Ferry, 1991 : 382), rend explicite cette idée de chaîne de danseurs dont la qualité première est d'être continue. Autrement dit, l'homokinésie (Beaudet, 2001) de chaque groupe de danseurs objective l'idéal de solidarité, d'unité organique qu'est censée incarner chaque classe d'âge.

Avant de commencer la danse, les hommes se réunissent aux abords immédiats du village avec leurs pères de classe d'âge okwetək $(\mathrm{F})$ dans un lieu abrité du regard des femmes. En consommant les quelques jarres de bière prévues pour cette réunion, chacun vérifie que ses ornements sont bien arrimés, parfois assisté par l'un de ses «pères » de classe d'âge. Arborant de grands cimiers de plumes de vautours, coiffés d'une longue bande blanche de coton tissé, des ceintures de perles et d'aluminium autour de la taille, des ornements de poils de moutons autour des biceps et de franges blanches aux avant-bras, les danseurs odyar (E) arrivent sur la place de 
danse en procession, devancés par leurs pères de classe d'âge okwetək (F) qui balancent doucement de leur main gauche un chasse-mouche en crins de cheval. Quelques hommes de la classe okwetzk $(\mathrm{F})$ se relaient pour jouer les tambours. Ce sont eux qui sont garants du tempo et imposent qu'il ne ralentisse pas. Les hommes odyar (E) maintiennent une pulsation isochrone à l'aide d'une cloche à battant externe (è-boyi) tenue dans une main ${ }^{4}$, tout en entretenant un fond sonore continu produit par une batterie de cloches agitée de l'autre main.

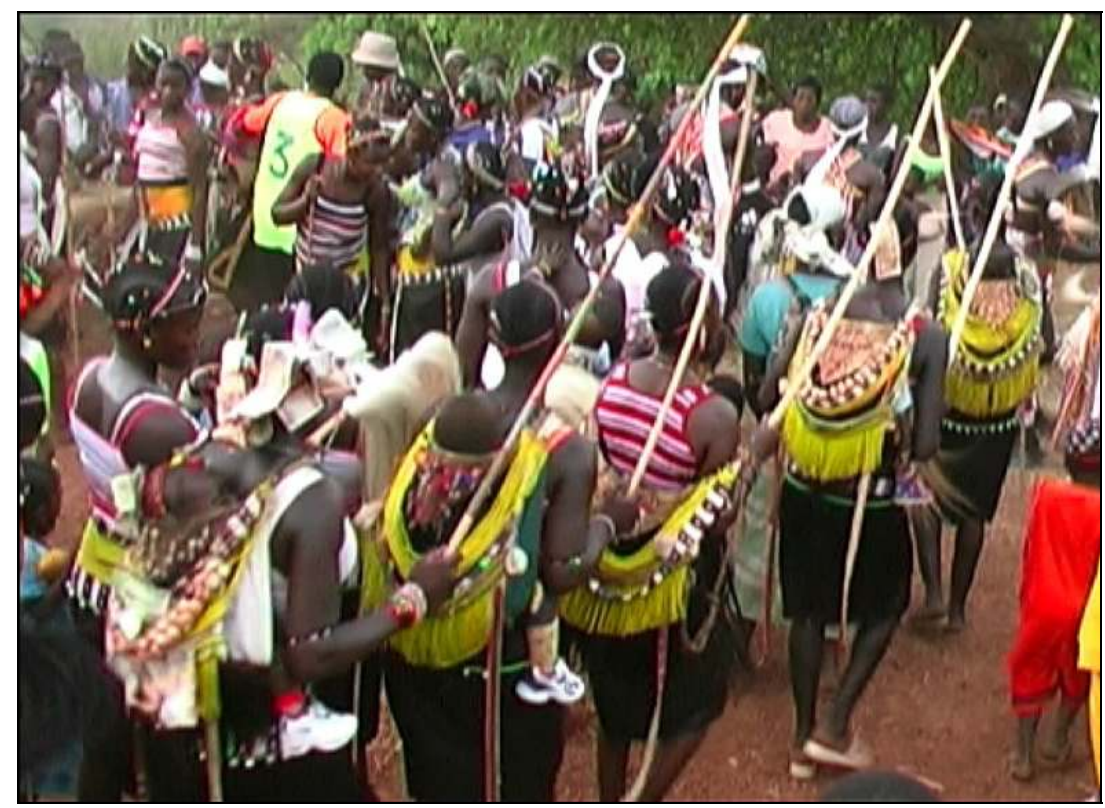

Photogramme 1. Danseuses od-obatyaya et od-odyar.

Le pas de danse des hommes est une variation de la marche avec un léger doublé du même pied et un faible temps de suspension (GGDDGGDDGGDD). La posture générale consiste à garder l'ensemble du corps relativement compact, le buste légèrement incliné vers l'avant. Cette posture combinée avec la marche cadencée produit un léger balancement de l'ensemble du corps de gauche à droite qui permet à son tour d'animer d'un mouvement oscillatoire toute une série d'ornements : d'un côté les franges perlées de leur ceinture ainsi qu'une sorte de queue constituée de lanières de cuir qu'ils portent au niveau des reins, de l'autre les extrémités du cimier en plumes, à savoir d'une part les boules de coton blanc qui prolongent les plumes et, d'autre part, une longue chaîne perlée qui, fixée sur le sommet du

\footnotetext{
${ }^{4}$ Le battant externe est un anneau fixé au pouce de la main qui tient la cloche.
} 
cimier, pend presque jusqu'au sol devant le danseur (cf. photogramme 2). La qualité du mouvement ondulatoire particulier de cette dernière chaîne est un marqueur clair de la qualité du mouvement dansé. A l'inverse de la posture générale contrainte, le relâchement musculaire doit être beaucoup plus grand pour animer les ornements qui sont fixés aux bras. Calés sur la même périodicité que le pas, les coudes font un léger mouvement de va-etvient l'un vers l'autre de telle sorte que les extrémités en poils de mouton des deux brassards dessinent idéalement un mouvement symétrique quasi circulaire. La tâche est d'autant moins aisée que les bras sont dans une position dissymétrique, le bras gauche qui tient la batterie de cloches est détendu alors que le bras droit qui tient la cloche à battant externe est replié. Malgré la tension musculaire - et parfois la douleur - qui permet de maintenir l'ostinato de la cloche à battant externe, le bras droit doit lui aussi produire un mouvement mesuré pour permettre le balancement de l'ornement de lanières de cuir (ou de plastique comme c'est plus courant aujourd'hui).

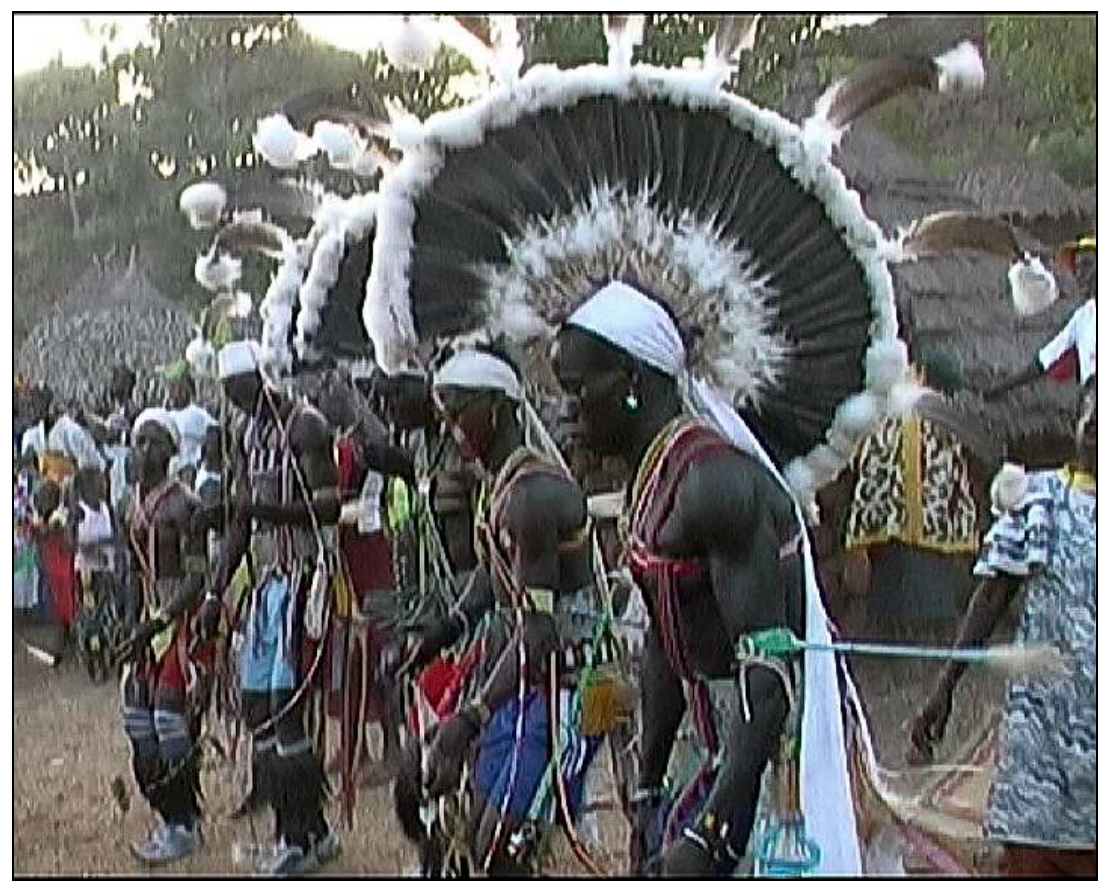

Photogramme 2. Danseurs odyar.

La précision du geste dansé aboutit à un mouvement ornemental régulier. Mais le rapport entre les deux s'établit de manière indirecte car la réponse ornementale au geste dansé est amplifiée par un effet purement 
mécanique, selon lequel la fréquence du mouvement de l'ornement se trouve dans un rapport double du geste qui en est à l'origine. A l'image d'un pendule qui n'a besoin que d'une impulsion pour se balancer deux fois, le pas d'un danseur à la noire engendre un balancement ornemental à la croche. C'est certainement cette non congruence des mouvements minimalistes et contrôlés du corps et de l'animation ample et fluide des ornements qui contribue le plus à produire un effet visuel et sonore global qui capture l'attention - au moins autant des danseurs que du public - en donnant l'illusion que ces appendices corporels sont auto-animés. Le «piège à pensée » (Smith 1979) de la danse réside dans la tension qu'introduit ce décalage perceptuel. En outre, si le mouvement corporel semble être conditionné par l'effet ornemental qu'il doit produire, le déplacement de la chaîne de danseurs renforce encore cette tendance. La chaîne de danseurs se déplace en effet dans un sens antihoraire, en alternant des phases stationnaires (sans s'arrêter de danser), orientées une fois vers la périphérie, une fois vers le centre, de telle sorte que le public dispose d'une double perspective, frontale et postérieure, sur l'ensemble des danseurs, et donc également sur leurs ornements.

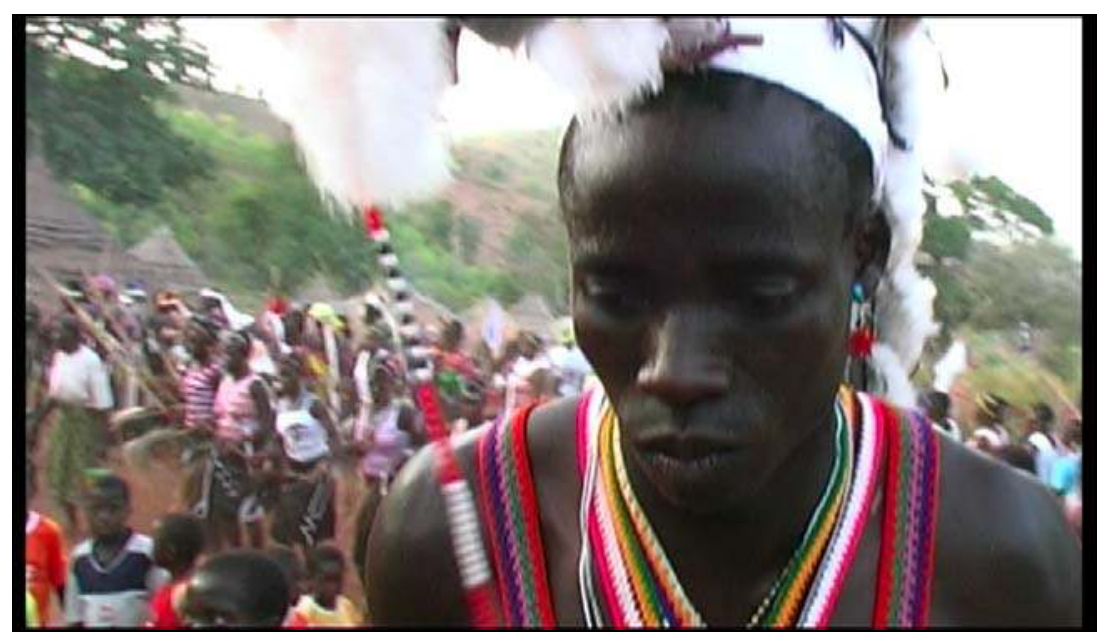

Photogramme 3. Danseur odyar.

Une fois le cercle des hommes installé, les femmes des deux classes intègrent la place de danse en formant deux cercles concentriques. Les «mères » od-obatyaya (F') se placent dans un cercle inférieur contenu par celui des hommes et les «filles » od-odyar (E') occupent un cercle plus petit encore, inclus dans les deux autres. Les od-obatyaya (F') sont comme leurs «filles » od-odyar (E') tressées de telle sorte que le postiche qui se situe sous la natte sagittale donne à leur crane une forme allongée. On 
retrouve donc l'amorce de ce qui pourrait être un cimier en miniature. Les «mères » $\mathrm{y}$ fichent des bijoux en aluminium décorés de pompons rouges à base de coton. L'ornement le plus subtilement décoré des femmes odobatyaya $\left(\mathrm{F}^{\prime}\right)$, en plus des ceintures de perles et des ornements de tête qu'elles arborent, est un porte-bébé ( $a$-ndənd), traditionnellement en peau de chèvre, mais aujourd'hui assez fréquemment en tissu, auquel est fixée une ceinture de perles jaunes analogue à celle des hommes odyar. Toutefois, l'ornement qui les distingue en étant la propriété exclusive de leur classe d'âge est le chasse-mouche fabriqué à partir de crins de cheval (a-ndyira) que possèdent également leurs homologues masculins okwetək $(\mathrm{F})$. Comme les hommes, elles le tiennent dans la main gauche en le balançant doucement de gauche à droite (cf. photo 4).

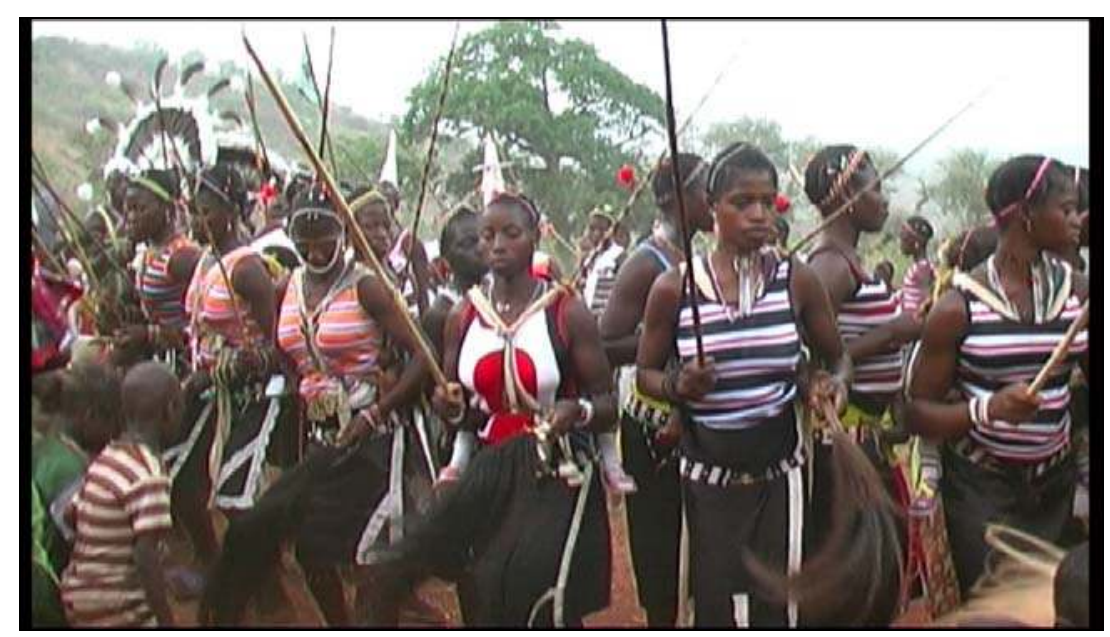

Photo 4. Danseuses od-obatyaya au premier plan et od-odyar en arrière plan.

Les deux groupes de femmes ont dans la main droite un bâton qu'elles tiennent devant elles vers le haut et dont le mouvement scande leur pas. $\mathrm{Au}$ sommet de ce bâton se trouve parfois sculptée en miniature l'effigie d'un danseur, clairement identifiable par sa crête sagittale et éventuellement des boucles d'oreilles. D'une manière générale, la parure des femmes paraît beaucoup plus modeste que celles des hommes mais, en ne considérant que les femmes, celle des «filles» est plus minimaliste encore que celle des «mères ». Aucun de leurs ornements ne possède la mobilité virtuelle des ornements masculins : les bandes de coton tissé (analogues à celles dont les hommes se coiffent) qu'elles portent en bandoulière comme les ceintures de perles qu'elles ont autour de la taille demeurent collées à leur corps. L'animation potentielle des ornements est réduite à sa plus simple expression. 
Ce caractère différentiel s'exprime avec davantage de netteté lorsqu'on observe leur pas de danse. Le pas des «mères » est dans un rapport homorythmique avec celui des hommes, suivant le même profil GDGDGDGD, mais son amplitude est plus réduite (il n'y a pas de temps de suspension). En revanche, le pas des «filles », pourtant lui aussi calqué sur la pulsation d'ensemble, semble se dérouler au ralenti. Par une opération très simple qui consiste à doubler le même pied (GGDDGGDD), leur pas de danse peut en effet paraître deux fois plus lent. La conséquence de cette impression de ralentissement n'est pas neutre car elle implique une réduction de moitié de la fréquence du déhanchement engendré par la marche alternée. Alors que les perles fixées au porte-bébé des «mères » conservent une partie de l'esthétique valorisée du balancement des ornements masculins, il n'en reste pour ainsi dire presque rien chez les «filles ». En outre, les pas de chacune de ces chaînes de danseurs produisent un effet ornemental dont le centre de gravité varie. Le pas réduit des filles n'anime véritablement que la partie la plus basse du corps, la cadence plus soutenue des mères est absorbée au niveau des hanches, et la rigidité du bassin des hommes transfère le mouvement dans les parties supérieures. Loin d'être aléatoire, les zones du corps en mouvement correspondent aux parties mobiles des ornements qui peuvent être soulignés, notamment les perles du porte-bébé des «mères » et les plumes $\mathrm{du}$ cimier des hommes. Au caractère minimaliste de l'ornementation des «filles» s'ajoute que, si leurs «mères» suivent la chorégraphie masculine en offrant comme eux au regard extérieur une double perspective (alternance de postures stationnaires dansées orientées vers l'intérieur et l'extérieur), les filles ne se présentent que sous l'angle latéral qu'autorise leur pas sans variation de direction.

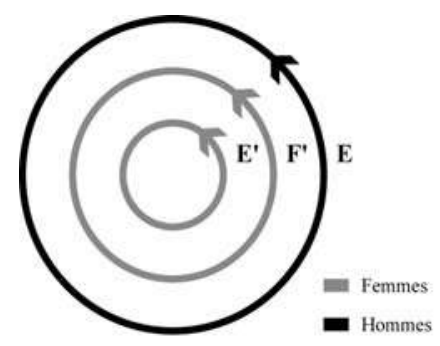

\begin{tabular}{l|l|l} 
Classe & Alternance du pas dansé & Echelle \\
\hline Odyar (E) & GdDgGdDgGdDgGdD & $1 / 1$ \\
\hline Od-obatyaya (F') & G D G D G D G D & $1 / 2$ \\
\hline Od-odyar (E') & G G D D G G D D & $1 / 4$
\end{tabular}

Fig. 3 - Chorégraphie concentrique et rapport d'échelle 
En d'autres termes, non seulement les mouvements prescrits ne sont pas équivalents d'une chaîne de danseurs à l'autre, mais tant du point de vue de la nature de leurs ornements, de celui de leur potentiel de mobilité, ou encore de la manière dont ils sont rendus visibles, tout concourt à différencier hiérarchiquement, dans un premier temps, les hommes des femmes, dans un second, les mères des filles. Selon une succession hiérarchique qui va des hommes aux femmes et des mères aux filles, on observe une diminution croissante de la liberté gestuelle corrélée à une ornementation de moins en moins saillante par la réduction qui s'opère tant en terme de nombre, que de mobilité intrinsèque ou encore de perspectives offertes au regard d'autrui (cf. figure 3 ).

Si l'on adopte le point de vue d'un spectateur, on observe la danse depuis l'extérieur en ayant les hommes odyar au premier plan, les femmes od-obatyaya au second plan, puis leurs filles od-odyar en arrière plan. Depuis cette perspective, chaque chaîne de danseurs semble être l'écho ornemental, la réitération estompée de celle qui se situe immédiatement à sa périphérie. Mais il ne s'agit pas seulement d'un phénomène d'altération progressive, car c'est cette opération qui donne à la chaîne périphérique profondeur et intensité. Pour le dire autrement, on peut prendre une métaphore acoustique et se représenter la danse dans son ensemble comme constituant un seul son. La chaîne de danseurs en serait alors la note fondamentale et les chaînes intérieures les fréquences harmoniques qui lui sont liées en tant que multiples constitutifs. Tout en appartenant à une même série dégressive du point de vue de son intensité, la richesse de la fondamentale masculine est indissociable de ses résonances harmoniques féminines. Il est possible de prolonger cette métaphore en l'appliquant à d'autres danses, telle que celle des olug (génération alterne des odyar), calibrée exactement sur le même modèle, mais aussi celle des opalug («fils » des odyar) dont le son particulier tient justement, pourrait-on dire, au fait qu'il n'a pas d'harmoniques féminines. Qu'il n'y ait pas de femmes dans cette dernière «danse d'hommes » est à rapprocher du fait que les opalug sont ceux qui, encore célibataires, tiennent le rôle le plus actif dans les brimades de l'initiation masculine, par opposition aux odyar, souvent déjà mariés et dont la participation initiatique remplit une fonction modératrice.

Puisque tout semble converger pour rendre saillants les ornements des danseurs, et ceux des hommes en premier lieu, on peut se demander les raisons d'une telle emphase. On pourrait même s'interroger sur ce qui, malgré la focalisation opérée sur les ornements masculins, fait de cette danse une "danse d'hommes », dans la mesure où davantage de femmes que d'hommes y participent. La prééminence masculine de la danse ow i est 
pourtant évidente et indiscutable pour les Bassari. En témoigne déjà assez simplement le fait qu'il est impensable que seules des femmes soient présentes sur la place de danse ; l'inverse n'étant pas valorisé mais possible. Mais pour comprendre les raisons plus profondes de cette polarité masculine, il faut la resituer dans la continuité des rites masculins de passage d'une classe d'âge à une autre, ritualisation dont elle est le prolongement chorégraphique.

\section{«LES BLANCHIS »}

Les ornements distinctifs de la classe des odyar (E), tels ceux de couleur blanche et la cloche métallique è-boyì, ne s'obtiennent qu'au terme de plusieurs cérémonies au cours desquelles les jeunes hommes opalug (D), à la fin de leur cycle et donc candidats au grade suivant, subissent une série de brimades initiatiques. Lors de l'hivernage qui précède l'année de changement, ils doivent effectuer collectivement vingt-six jours de culture (emáyi), qu'on décompte sur une corde nouée (chaque nœud représentant une journée de travail), afin d'obtenir des familles dans lesquelles ils ont travaillé suffisamment de mil pour offrir la bière que boiront leurs classes aînées l'année suivante. Pendant la dernière initiation d'un cycle de six ans, l'année de changement donc, les opalug quittent le village pour aller passer la nuit en brousse. Au matin, commence la cérémonie ekwes ${ }^{y}$. Ils sont enduits de kaolin, puis exposés immobiles au soleil pendant plusieurs heures. Après avoir été littéralement «mis à sécher», ils doivent lutter, affaiblis, avec leurs pères de classe d'âge. Ces derniers détruisent ensuite leur maison d'âge (ambofor) - plus tard reconstruite par les initiés en passe de devenir olug (B) - transformant la paille du toit de la maison saccagée en tisons avec lesquels ils poursuivent leurs «fils » en simulant de leur brûler le dos.

Quinze jours plus tard, pendant la cérémonie imana interdite aux femmes, ils reçoivent de deux masques lukwuta ${ }^{5}$, choisis parmi la classe des odyar, quatre coups de fouet - deux par masque - devant laisser des cicatrices sur l'arrière des bras. Ce sont les plaies non cicatrisées de cette épreuve, nommée è-kapa (litt. «l'attache»), qu'affichent fièrement les hommes lorsqu'ils dansent pour la première fois ow wi la semaine suivante à l'occasion d'ipèsyan (litt. «Les blanchis »). Il est tentant d'envisager ces marques corporelles comme une forme limite de l'ornementation, lesquelles apparaissent d'ailleurs d'autant plus mises en avant que les danseurs n'ont pour cette première occasion pas encore le droit de porter les captivants

\footnotetext{
${ }^{5}$ Comme les opalug lors de la cérémonie précédente, les masques sont «blanchis », mais à partir d'un mélange de terre et de cendre.
} 
cimiers en plumes de vautour. C'est à l'issue de cette épreuve que les hommes gagnent le droit de porter leurs ornements distinctifs, notamment des ceintures de perles blanches autrefois faites de cauris, la bande blanche de coton tissé et la cloche è-boyì. Au moment même de l'exécution de la première danse, le changement de classe est effectif et irrémédiable : les opalug ont pris la place des odyar, les odyar sont devenus okwetzk (litt. « les finis », « les terminés »), les okwetzk remplacent les opidor, etc.

A partir de cette description très succincte du changement de classe d'âge, il apparaît clairement qu'en affichant de manière ostentatoire leurs ornements dans la danse ow wi, les hommes activent un symbolisme largement extérieur à la danse, lui-même engendré au cours de cette longue série d'épreuves initiatiques. La continuité chromatique qui peut être établie entre le «blanc» des épreuves (celui des corps enduits de kaolin de la première cérémonie et des masques de la seconde) et le «blanc» des ornements est un moyen d'évoquer la résistance aux souffrances infligées par leurs «pères » de classe d'âge. Les chants que les hommes entonnent la nuit, lorsqu'ils font par petit groupe le tour des cases pour répondre aux invitations de nourriture et de bière de leurs «amies » (nyapra), évoquent également, de manière plus ou moins voilée, le déroulement de ces cérémonies pourtant interdites aux femmes :

Alors qu'on dit qu'ekapa est une joie,

Ils ont voulu me tuer.

Tuez- moi!

Avec mes nouvelles perles blanches [cauris].

Le raccourci que ce chant opère entre les brimades initiatiques des masques et les ornements distinctifs de leur classe d'âge est saisissant. Dans le même temps que les hommes chantent leur nouveau statut (les perles blanches), ils rappellent leur courage d'avoir surmonté l'épreuve des coups infligés par les masques (ils ont voulu me tuer). Rappelons au passage qu'il ne saurait pourtant être question pour les nouveaux odyar d'afficher dans la danse une attitude triomphante face à cette victoire initiatique ou quant au fait d'avoir poussé leurs «pères» dans une classe moins faste ${ }^{6}$. Un visage impassible et ne laissant transparaitre aucune émotion est de rigueur. Le changement de classe actualise une relation qui reste stable dans sa forme

\footnotetext{
${ }^{6}$ Contrairement à la classe des okwetek qui ne jouit d'aucun prérogative particulière, la classe des odyar est en effet unanimement désignée comme la classe masculine par excellence (les exégèses étymologiques sont d'ailleurs très pauvres puisqu'on parle simplement d'eux comme «les hommes »). Classe de ceux dont le comportement est censé être juste et responsable, ils sont chargés d'enterrer les morts, de partager la bière et la viande ; parmi eux encore sont choisis les « garants de la coutume » (Gaməma).
} 
en ce sens que les « fils » conservent une position subordonnée par rapport à leurs «pères ». Un autre exemple de chant illustre encore, quoique de manière plus cryptée, cette correspondance entre violence subie et ornement. Les hommes chantent :

Ecoutez cela :

N'y a-t-il pas un seul odyar dans votre village?

Les jeunes, venez sonner la cloche.

Ce chant reste obscur sans le contexte de sa création. En 2008, un des villages de l'aire 6ə-xoré décida d'annuler les coups donnés lors d'ekapa. Dans un autre village, où les flagellations étaient encore en vigueur, un homme odyar composa ce chant, dont l'intention moqueuse est claire. On peut le paraphraser ainsi : "Vous qui n'avez pas véritablement enduré ekapa, qui pourra jouer la cloche è-boyì sinon des jeunes ${ }^{7}$ ? ». L'insistance des hommes à réinstaurer la distinction de sexe en rappelant aux femmes leur exclusion du contenu secret des cérémonies n'épuise pas les interprétations qu'on peut faire de la place qu'occupent les femmes dans cette danse. Elles sont loin de n'être que les destinataires passives des secrets exhibés des hommes. Pour les od-obatyaya, on peut déjà voir qu'elles instaurent avec leurs filles od-odyar, sur le plan chorégraphique, une modalité de relation qui, dans une certaine mesure, prend une forme analogue à celle que les hommes entretiennent entre eux sur le plan initiatique. Dominées par leurs «mères» dans la vie quotidienne (un nombre considérable d'amendes payables en journées de travail leur est régulièrement imposé), les od-odyar le sont également dans la danse. La «formalisation» (cf. Bloch 1974) de la danse ow wi réduit les mouvements des od-odyar à une expression minimale et leur interdit, contrairement aux od-obatyaya, de répondre aux chants des hommes. Les relations de subordination de sexe parallèle ${ }^{8}$ entre les femmes apparaissent comme la projection, sur le plan chorégraphique, des relations violentes de sexe parallèle entre les hommes sur le plan initiatique. L'homologie qui existe entre ces deux classes d'hommes et ces deux classes de femmes est bien manifeste car la subordination des «fils » aux «pères » reste, comme celle des «filles » à leurs «mères », visible dans la danse. La domination des «pères » vis-à-vis de leurs « fils » reste saisissable aussi bien par le fait que

\footnotetext{
${ }^{7}$ Le terme qu'on emploie dans le chant, otyambendyar, qu'on peut traduire par «jeunes », désigne en effet les deux classes $\mathrm{C}$ et $\mathrm{D}$, par opposition aux «hommes » (classes $\mathrm{E}$ et suivantes) et aux «non-initiés » (classes A et $\mathrm{B})$.

${ }^{8}$ Sexe parallèle est ici entendu comme la traduction du terme same-sex proposé par Marilyn Strathern (1988). Il désigne les relations entre personnes de même sexe (H/H ou F/F), par opposition au terme cross-sex, que je traduirai par sexe croisé, qui s'applique aux relations entre personnes de sexe différent $(\mathrm{H} / \mathrm{F}$ ou $\mathrm{F} / \mathrm{H})$.
} 
les seconds se plient au tempo qu'imposent les premiers que par la possibilité de se relayer dans un contexte où la performance est unanimement perçue comme pénible et éprouvante (toute la classe des «pères » participe mais par groupes successifs de seulement deux ou trois tambourinaires).

En poursuivant l'origine des ornements, on bascule rapidement dans un registre qui ne se limite pas aux seules relations de sexe parallèle. Les bâtons de danse que les femmes tiennent dans la main sont par exemple typiquement des cadeaux que se font les garçons et filles qui sont liés en tant que nyapra. Ces bâtons de danse sont les indices de relations d'échange très codifiées qui existent dans le cadre de l'amitié formelle des hommes et des femmes d'une même maison cérémonielle et d'un même groupe d'âge. Pendant oxi, les femmes od-odyar offrent à leur nyapra $\left(\mathrm{E}^{\prime} \rightarrow \mathrm{E}\right)$ une nourriture choisie (plats de riz et poulets) qui est elle-même le contre-don des cadeaux (argent, part de gibier) que ces mêmes hommes, lors de leur appartenance à la classe d'âge précédente, leur offraient $\left(D \rightarrow D^{\prime}\right)$, notamment pendant l'initiation. Par ailleurs, les ceintures de perles que portent les danseurs sont typiquement une production féminine, ce qui suppose donc que les hommes sollicitent les femmes de leur entourage pour parvenir à se les procurer.

La plupart des ornements masculins, qu'ils soient fabriqués par les hommes ou les femmes, existent en stock limité. Se déploie donc tout un jeu stratégique d'emprunts pour obtenir d'une sœur ou d'une mère telle ceinture de perles, d'un beau-frère ou d'un oncle maternel le rare cimier de plumes, d'un voisin ou d'un agnat la cloche métallique, etc. Par la complétude des ornements que porte un homme, on peut déduire sa capacité à mobiliser tout un ensemble de relations (affinité, consanguinité, corésidence) relevant de la compétence qu'on attend d'un sujet masculin. L'inverse est également vrai. L'aspect dénudé d'un danseur qui n'a pas réussi à collecter au complet ses ornements est le signe d'un isolement relationnel, donc d'une certaine forme de marginalité.

Mais dans le cadre formel engendré par la danse, cette incitation à l'exhaustivité relationnelle se réduit drastiquement à une opposition entre, d'une part, des relations de sexe croisé symétriques fondées sur une logique d'échange (les nyapra) et, d'autre part, des relations de sexe parallèle asymétriques informées par des positions hiérarchiques (les «pères » et les «fils»; les «mères » et les « filles »). Le lien d'affinité potentielle (et parfois réelle) entre mbanyira, est ainsi passible de deux définitions pour chacune des perspectives masculines et féminines. Pour un ego masculin, la classe des femmes qu'il appelle mbanyira représente à la fois la classe de celles qui englobent hiérarchiquement ses partenaires d'échange («mères » 
des nyapra) et la classe de celles qui échangent avec ceux qui l'englobent hiérarchiquement (nyapra des "pères »). Réciproquement, pour un ego féminin, l'époux potentiel (ou réel) est alternativement le nyapra d'une «fille» ou le «fils» d'un nyapra. Il est frappant de remarquer que la relation entre mbanyira ( « ceux qui dansent ensemble»), en tant que telle, n'est pas qualifiée et reste largement indéterminée. Elle apparaît plutôt comme le produit émergent de deux chemins alternatifs impliquant chacun la composition de deux modalités de relations systématiquement complémentaires, du point de vue de l'affiliation résidentielle (même ambofor/ambofor différent), des rapports entre classe d'âge (identique/consécutif), des relations de genre (parallèle/croisé). La figure 4 ci-dessous l'exprime de manière graphique :

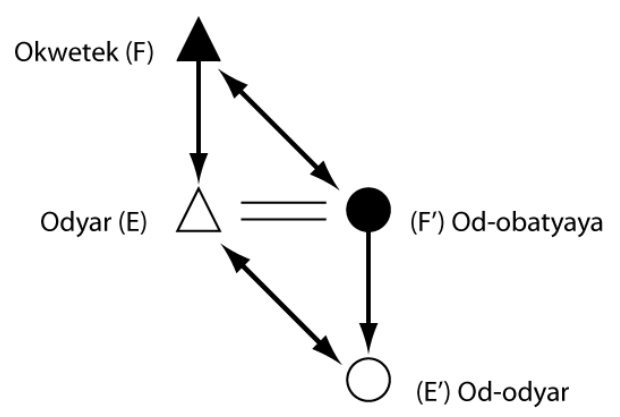

Fig. 4 - Contexte formel de la relation mbanyra

Bien que ce qui compte surtout dans la danse $o \tilde{w i}$ est la manière dont les hommes exhibent leurs ornements comme marqueurs sociologiques de leur appartenance d'âge - donc comme les indices des relations violentes des «pères» de classe d'âge à l'égard de leurs «fils»-, ils restent doublement dépendants des femmes. Ils sont liés aux femmes de leur classe d'âge par des relations électives d'échange ( «les bâtons des nyapra»), mais aussi à leurs mbanyira par des relations virtuelles de mariage. Ceci apparaît très clairement lorsqu'on regarde l'ornement des od-obatyaya que la danse souligne. Le porte-bébé que les femmes n'ont de cesse de décorer et que leur pas de danse anime est par excellence l'indice d'une relation d'alliance. En effet, la compensation matrimoniale qu'un homme verse à ses beauxparents et à l'oncle maternel de sa femme comporte toujours, parmi les six ou huit chèvres dont c'est généralement le montant, une dont on dit qu'elle est « celle du porte-bébé ». Généralement versée à la mère de l'épouse, cette chèvre est parfois transmise à une sœur qui la restituera au moment du mariage d'une de ses filles (Ferry \& Guignard, 1984 : 53). En arrière plan 
des hommes, les femmes od-obatyaya évoquent donc par leur porte-bébé, plus qu'une relation de mariage, la moitié d'une relation d'alliance, à savoir celle d'un homme avec les parents de sa femme, et tout particulièrement avec sa belle-mère. L'autre moitié de la relation d'alliance est donnée par l'explication, formidablement simple, que les Bassari donnent de la voix de poitrine, grave et gutturale, qui caractérise le chant des od-obatyaya, dont le nom de classe, rappelons-le, est dérivé. Il est explicitement dit de ce dernier qu'il s'adresse aux parents du mari, donc aux beaux-parents de la femme qui chante (Ferry, 1991 : 376). D'une manière extraordinairement synthétique sont exhibées, visuellement et vocalement, deux modalités élémentaires du lien d'alliance, la relation de chacun des conjoints avec ses beaux-parents respectifs.

En examinant le réseau d'intentionnalités que les ornements des danseurs et danseuses canalise, il est possible de dégager la forme relationnelle sous jacente à la danse. Selon la perspective qu'imposent les hommes, les chaînes collectives de danseurs apparaissent comme des groupes initiatiques; selon celles des femmes ce sont des partenaires d'échange. Autrement dit, la présupposition sociocentrée - induite par les hommes - d'une unité formelle rigoureuse (l'idée de «classe »), se trouve systématiquement contrecarrée par l'individuation - induite par les femmes - résultant de la réintégration des hommes à un réseau relationnel égocentré. $\mathrm{Au}$ croisement de ces deux perspectives, le lien d'alliance virtuelle apparait comme une combinaison singulière qui emprunte aux deux registres, tout à la fois relation classificatoire et relation prototypique d'échange.

Mais il est crucial de souligner que la forme relationnelle qui émerge de l'organisation collective de la danse ne traduit qu'une image particulièrement stéréotypée des relations entre les sexes et les âges. La concentration des groupes d'âges en un même lieu ainsi que leur engagement dans une action collective rigoureusement synchronisée constituent le mode privilégié par lequel le système d'âge est rendu publiquement visible dans un environnement où la dispersion maximale autorise rarement une vue d'ensemble. Ce faisant, la danse présente une sorte de modèle réduit des relations que suppose l'institution et où, à la formalisation extrême de la liberté gestuelle de la vie quotidienne correspond, par un effet de convergence, une épuration relationnelle qui ne laisse pas plus de place à l'improvisation qu'à l'expression des affects. La réserve émotionnelle des danseurs est en effet étroitement associée à une réduction radicale des possibilités d'interaction. Pour ne prendre qu'un exemple, il est rigoureusement impossible de distinguer, dans la danse ellemême, ceux qui parmi les couples potentiels sont véritablement mariés. Lorsque les mbanyira se font face, ce qui arrive périodiquement par la 
variation d'orientation des chaînes de danseurs, chacun détourne le regard. La danse donne à voir une version stylisée du mariage mais certainement pas une réalisation particulière. On pourrait en dire autant des autres relations qui doivent certainement une partie de leur force expressive à l'anonymisation que suppose la mise en acte de l'institution sous cette forme particulièrement condensée.

\section{OBJETS ANIMES ET IDENTITES COLLECTIVES}

Ne prendre en considération, comme je l'ai fait ici, qu'une seule danse, de surcroit fortement connotée comme étant une "danse d'hommes", introduit un biais majeur dans la compréhension que l'on avoir du système global des danses Bassari. En effet, il existe à côté de cette danse, non seulement d'autres danses masculines, mais aussi toute une série de danses féminines dont la prise en compte est absolument nécessaire pour ne pas restituer une image partielle et tronquée des principes qui organisent la systématique d'ensemble. Chaque danse devrait idéalement être recontextualisée dans le temps structural du système d'âge et être envisagée comme la transformation de celles dont elle est le prolongement ou l'anticipation. Cependant, la danse ow wi présente déjà, malgré son évidente valence masculine, suffisamment d'éléments pour tirer quelques conclusions provisoires.

Tout d'abord, la fonction de l'ornementation n'est absolument pas de souligner la singularité des personnes en fonction de leur corps. Tout le travail qu'opèrent les Bassari par le biais de l'ornementation œuvre au contraire à désingulariser les individus en abolissant leurs différences corporelles. L'effet combiné de l'unisson gestuel (Beaudet, 2001 : 61) et du caractère instrumental des corps qui sous-tendent l'esthétique de la danse est en effet ce qui tend à rendre les corps des danseurs indiscernables les uns des autres. La forme limite que prend cette logique d'indifférenciation poussée à son terme serait donc davantage celle d'un « corps collectif » que celle d'une collection d'individus. Il s'agit en effet moins d'agréger des individus déjà singularisés que de les désingulariser pour les faire apparaître comme un collectif indifférencié. On appréhende assez intuitivement la force d'un tel procédé pour faire émerger l'idée de groupe, les ornements jouant d'autant plus efficacement leur fonction d'affiliation sociologique qu'ils contribuent, en accrochant le regard, à atténuer au maximum les différences internes qu'on pourrait déceler au niveau des corps.

Sous cet aspect, l'ornementation bassari remplit une fonction quasi inverse de celle qu'exerce la peinture faciale des Jivaro telle que la décrit A.-C. Taylor (2006). Pierre de touche d'un individualisme radical, les motifs peints dont les Jivaro s'ornent le visage constituent l'indice d'une 
configuration relationnelle complexe qui, en combinant deux modalités de relations agonistiques (entre des égaux masculins et entre un vivant et un mort), a pour effet de différencier à l'extrême des sujets dont les corps, et tout particulièrement les visages, ne seraient pas forcément au départ suffisamment singularisés. Alors que la peinture corporelle des Jivaro semble être le moyen de parachever l'individuation des corps afin d'affirmer une idéologie individualiste, l'ornementation des Bassari semble au contraire être un moyen de neutraliser les différences corporelles pour faire apparaître des groupes. Mais, comme l'attestent les stratégies visant à arborer le costume le plus complet, la mise en place de cette identité collective ne se fait pas au prix d'une rupture des relations particularisantes. La complétude ornementale est au contraire le signe patent de la singularité qui résulte d'être connecté à des personnes avec lesquelles il n'existe pas uniquement des rapports formels comme ceux qui lient entre eux les différents groupes d'âge, mais aussi des relations vécues, marquées par le caractère négociable de la vie quotidienne et relevant des compétences stratégiques de sujets individualisés.

Un deuxième exemple peut servir à cerner par contraste ce qui se joue dans la danse bassari. A plusieurs reprises, les Bassari m'ont proposé une image intéressante en mettant en parallèle leurs pratiques et celles de l'armée : recrutement sur le seul critère d'âge et de sexe ; imposition d'un dressage des corps qui tend à les uniformiser ; grades et promotions aisément repérables par des signes distinctifs, etc. L'image est séduisante jusqu'à un certain point. Je crois en l'occurrence qu'elle est trompeuse et que les Bassari négligent un aspect fondamental. A l'inverse des jeunes recrues de l'armée qui sont par principe des individus déconnectés, les Bassari sont déjà tous en relation les uns avec les autres. Chacun dispose sur chacun de plusieurs modes d'identification fondés sur la parenté, la corésidence, l'amitié, la participation aux mêmes «groupes de travail» (andiana), etc. Bref, ils participent depuis le plus jeune âge à un univers relativement endogame déjà largement saturé d'interactions. Par conséquent, le travail rituel qui modèle ces relations à différentes étapes du cycle de vie des hommes et des femmes, à l'inverse de l'armée, vise moins à tenter de s'affranchir de ces liens qu'à les actualiser en établissant des correspondances systématiques entre eux : initiation et mariage (Ferry, 1985), classes d'âge et parenté. A l'opposé d'un défilé militaire, la danse n'exhibe pas la négation des relations de la vie quotidienne, mais plutôt l'actualisation expressive et stylisée de la manière dont elles sont rituellement infléchies. Les ornements sont les indices de ce travail ; la précision du mouvement qui les anime une ressource pour en apprécier l'efficacité. 
Au fil de cet article, le terme «ornement» a été entendu par défaut comme tous les objets, fabriqués et nommés, utilisés dans le cadre cérémoniel des danses. J'ai donc inclus aussi bien ce qui relève des parures corporelles que des instruments de musique, partant du fait que tous obéissent à des critères d'usage stricts dépendant de l'âge, du sexe et du contexte. En regroupant dans un même ensemble des artefacts que l'on rattache conventionnellement à des catégories distinctes (i.e. instruments de musique $v s$ parures corporelles), je n'ai fait que suivre les rapprochements que les Bassari eux-mêmes opèrent ${ }^{9}$. Il est courant par exemple de caractériser un ornement apparemment essentiellement visuel par l'onomatopée censée reproduire le son causé par sa mise en mouvement, qu'il s'agisse de l'entrechoquement de ses parties constitutives ou plus simplement du son produit par son frottement dans l'air. C'est le cas notamment de l'ensemble des «ornements pendants » (franges, lanières, chaînes de perles, etc.), désignés par le terme générique bə-pété-fété (Ferry, 1991 : 407), dont on retient autant la qualité visuelle que sonore. Les instruments de musique sont bien sûr eux aussi susceptibles d'être caractérisés par leurs propriétés sonores (les sonnailles qu'on attache au dessus du genou ou la taille appartiennent à la classe de «ceux qu'on entend»), mais il n'est pas moins fréquent d'insister sur le mouvement qu'ils supposent (une cloche peut être nommée par le mouvement spécifique qui la fait sonner en fermant la main). Qu'il s'agisse donc d'un idiophone ou d'un ornement, la frontière intuitive entre artefact visuel et artefact sonore est particulièrement poreuse car ce dont il est question est d'établir un rapport entre, d'une part, un type de geste qui provoque un mouvement et, d'autre part, la qualité sonore et visuelle particulière qui en résulte. Or, j'ai le sentiment qu'une labilité comparable s'applique à la distinction entre corps et artefacts lors de leur mise en mouvement dans la danse. Du flou interprétatif provoqué par ce brouillage des frontières résulte sans doute la tentation de réduire les corps à une fonction instrumentale ou, à l'inverse, de percevoir les ornements comme « auto-animés ».

C'est à ce niveau que je serais tenté d'identifier la marque spécifique des dispositifs chorégraphiques bassari, au travers de la manière dont certaines distinctions conventionnelles telles que celles entre ornements et instruments, ou entre corps et artefacts, sont sous-évaluées. Cela permet à la danse d'offrir aux participants l'expérience simultanée de deux modes d'identification radicalement différents, l'un relevant de l'indifférenciation collective des groupes d'âge initiatiques, l'autre de la place stratégiquement

\footnotetext{
9 En suivant la thèse défendue par Tim Ingold (2007), on pourrait soutenir l'argument inverse, à savoir que c'est moins un rapprochement des Bassari qu'une distinction propre à l'Occident.
} 
négociée au sein de réseaux relationnels égocentrés. Alors même que la saillance ornementale qui permet la neutralisation des différences corporelles autorise à hiérarchiser entre les catégories d'âge mais pas en leurs sein, la complétude ornementale reste le marqueur d'une densité relationnelle singularisante selon laquelle il est possible d'affirmer qu'untel détient ceci de telle personne, cela de telle autre, etc. Finalement, l'organisation formelle de la danse suggère un rapprochement qui ne fait qu'organiser en un seul évènement ce à quoi tout le monde a accès de manière disjointe, chacun étant dans la situation virtuellement conflictuelle de devoir afficher une solidarité indéfectible à un groupe d'âge qui courtcircuite pourtant complètement l'ensemble de relations électives ancrées dans la parenté, la corésidence ou encore l'amitié qui sont le lot commun de la vie sociale. C'est dans la possibilité de cette double interprétation potentiellement contradictoire, et plus encore dans le fait que la danse parvient à l'imposer de manière synthétique comme deux aspects indissociables d'une même réalité, que je suis tenté de voir l'aspect proprement génératif de cette danse. Ou pour le dire autrement, quelque chose que la danse fait mieux que d'autres activités sociales. Lors de ces grands rassemblements de danse, il s'agit tout autant de célébrer le système d'âge pour lui-même, en tant qu'institution qui règle les conduites et définit des normes relationnelles, que de laisser transparaître le processus par lequel il y parvient.

\section{Références Bibliographiques}

Beaudet, J.-M., 2001, Le lien. Sur une danse des Wayãpi, Protée, 29: 59-66.

BLOCH, M. 1974, Symbols, song, dance and features of articulation: Is religion an extreme form of traditional authority?, Archives européennes de sociologie, 15 : 55-81.

DehouX, V. \& M. Gessain, 1992, La musique bassari : un parcours obligé, Cahiers de Musiques Traditionnelles, 5: 17-35.

FERRY, M.-P., 1985, Mariage des femmes et initiation des hommes, Journal de la Société des Africanistes, 55 (1-2): 75-83.

—, 1991, Thésaurus Tenda. Dictionnaire ethnolinguistique de langues sénégaloguinéennes (Bassari, Bedik, Konyagi), Paris, Peeters [3volumes, 1280 p.].

FERry, M.-P. \& Guignard, E., 1984, Tiges de mil, tiges d'igname. Essai sur la parenté chez les Bedik et les Beliyan (Sénégal oriental), L'Homme, XXIV (3-4): 35-60.

Gell, A., 1998, Art and Agency. An Anthropological Theory, Oxford, Clarendon Press.

GESSAIN, M., 1971, Les classes d'âge chez les Bassari d'Etyolo (Sénégal Oriental), in D. Paulme (éd.), Classes et associations d'âge en Afrique de l'Ouest, Paris, Plon: $157-184$.

—, 2006, La femme et le masque ou l'éloge de l'équilibre chez les Bassari, Paris, Sepia. 
Houseman, M., 2006, Relationality, in J. Kreinath, J. Snoek et M. Stausberg (éds.), Theorizing rituals. Issues, Topics, Approaches, Concepts, Annotated Bibliography, Leiden, Brill: 413-428.

Houseman, M. \& C. SEVERI,..1994, Naven ou le donner à voir. Essai d'interprétation de l'action rituelle, Paris, CNRS Éditions-Éditions de la Maison des sciences de l'homme.

IngOLD, T., 2007, Lines. A Brief History, London \& New York, Routledge.

Lestrange, M.-T. de \& M. Gessain, 1976, "Collections Bassari du Musée de l'Homme, du Département d'anthropologie de l'Université de Montréal, du Musée de l'IFAN à Dakar, et du CRDS à Saint-Louis (Sénégal)", Objets et Mondes, 15: 5-337.

Strathern, M., 1988, The Gender of the Gift. Problems with Women and Problems with Society in Melanesia, Berkeley/Los Angeles/Oxford, University of California Press.

TAYLOR, A. C., 2003, Les masques de la mémoire. Essai sur la fonction des peintures corporelles jivaro, L'Homme, 165: 223-248. 